\title{
Genetic and pharmacologic blockade of central melanocortin signaling attenuates cardiac cachexia in rodent models of heart failure
}

\author{
Jarrad M Scarlett, Darren D Bowe, Xinxia Zhu, Ayesha K Batra, Wilmon F Grant and Daniel L Marks \\ Department of Pediatrics, Oregon Health and Science University, Mailcode L481, 3181 SW Sam Jackson Park Road, Portland, Oregon 97239, USA \\ (Correspondence should be addressed to D L Marks; Email: marksd@ohsu.edu)
}

\begin{abstract}
The central melanocortin system plays a key role in the regulation of food intake and energy homeostasis. We investigated whether genetic or pharmacologic blockade of central melanocortin signaling attenuates cardiac cachexia in mice and rats with heart failure. Permanent ligation of the left coronary artery (myocardial infarction (MI)) or sham operation was performed in wild-type (WT) or melanocortin-4 receptor (MC4R) knockout mice. Eight weeks after surgery, WT-Sham mice had significant increases in lean body mass (LBM; $P<0 \cdot 05)$ and fat mass $(P<0 \cdot 05)$, whereas WT-MI did not gain significant amounts of LBM or fat mass. Resting basal metabolic rate (BMR) was significantly lower in WT-Sham mice compared to WT-MI mice $(P<0 \cdot 001)$. In contrast, both MC4-Sham and MC4-MI mice gained significant amounts of LBM $(P<0 \cdot 05)$ and fat

mass $(P<0 \cdot 05)$ over the study period. There was no significant difference in the BMR between MC4-Sham and MC4-MI mice. In the second experiment, rats received aortic bands or sham operations, and after recovery received i.c.v. injections of either artificial cerebrospinal fluid (aCSF) or the melanocortin antagonist agouti-related protein (AGRP) for 2 weeks. Banded rats receiving AGRP gained significant amount of LBM $(P<0 \cdot 05)$ and fat mass $(P<0 \cdot 05)$ over the treatment period, whereas banded rats receiving aCSF did not gain significant amounts of LBM or fat mass. These results demonstrated that genetic and pharmacologic blockade of melanocortin signaling attenuated the metabolic manifestations of cardiac cachexia in murine and rat models of heart failure.

Journal of Endocrinology (2010) 206, 121-130
\end{abstract}

\section{Introduction}

Chronic heart failure (CHF) is a major public health problem in western countries, with an incidence approaching 10 per 1000 population per year in those aged 65 years or older (Cowie et al. 1999, Lloyd-Jones et al. 2002). In the United States, CHF contributed to 284365 deaths in 2004, and the estimated direct and indirect health care costs to patients with CHF for 2008 were $\$ 34 \cdot 8$ billion (Rosamond et al. 2008). The prognosis of CHF patients is significantly worsened by the development of cachexia, a wasting condition that also manifests itself in other chronic illnesses, including cancer, chronic renal failure, AIDS, and inflammatory bowel disease (Tisdale 1997). Cardiac cachexia is a complex metabolic disorder involving features of anorexia, catabolism of protein and fat, reductions in bone mineral density, activation of acute phase response, and insulin resistance (Tisdale 1997). CHF patients that develop cardiac cachexia have increased morbidity and mortality compared to CHF patients who do not become cachectic, an effect that is independent of left ventricular function (Anker et al. 1997). Indeed, one study demonstrated that the mortality at 18 months in CHF patients that had been diagnosed with cardiac cachexia was as high as
$50 \%$ compared with $17 \%$ in patients with CHF that did not develop cardiac cachexia (Anker et al. 1997).

Although the exact molecular mechanisms that initiate and maintain the cachectic state in CHF remain largely unresolved, there is increasing evidence to suggest that increased production of proinflammatory factors including cytokines, chemokines, and eicosanoids may play a crucial role in the pathogenesis of cardiac cachexia. Specifically, there has been an increased focus placed on the potential role of proinflammatory cytokines in the pathogenesis of cardiac cachexia, as elevated serum levels of tumor necrosis factor- $\alpha$ (TNF- $\alpha$ ), interleukin-1 $\beta$ (IL-1 $\beta$ ), and IL-6 have been measured in CHF patients with cardiac cachexia (Levine et al. 1990, Dutka et al. 1993, Anker et al. 1999). Administration of proinflammatory cytokines, either peripherally or centrally, induces anorexia, weight loss, and activation of the hypothalamic-pituitary-adrenal axis (Plata-Salaman et al. 1996, Plata-Salaman 2001). The metabolic and behavioral responses elicited by proinflammatory cytokines are hypothesized to occur via their interaction with circuits in the central nervous system that regulate metabolism and food intake (Campbell 1998, Reyes \& Sawchenko 2002). 
A candidate target for inflammatory cytokines in the brain is the central melanocortin system. The central melanocortin system plays a critical role in the regulation of feeding behavior, linear growth, and metabolic rate (Baskin et al. 1999, Cone et al. 2001). This occurs principally via the action of $\boldsymbol{\alpha}$-melanocyte-stimulating hormone $(\boldsymbol{\alpha}-\mathrm{MSH})$, a peptide derived from the proopiomelanocortin (POMC)-expressing neurons in the hypothalamus and brainstem (Cone 2005). The feeding and metabolic effects of $\boldsymbol{\alpha}-\mathrm{MSH}$ are mediated by the melanocortin-4 receptor (MC4R), a G protein-coupled receptor expressed predominately in the brain (Cone 2005). Acute and chronic stimulation of MC4R produces anorexia, weight loss, and increased metabolic rate (Fan et al. 1997, Foster et al. 2003), whereas genetic deletion or antagonism of MC4R by agouti-related protein (AGRP), an endogenous melanocortin receptor inverse agonist, promotes food intake and weight gain (Ollmann et al. 1997, Joppa et al. 2005). Recent studies have shown that the secretion of $\alpha-\mathrm{MSH}$ is increased and the secretion of AGRP is decreased from hypothalamic explants in response to IL1 $\beta$ (Scarlett et al. 2007, 2008), demonstrating that central melanocortin signaling can be increased in response to an inflammatory cytokine.

Data from a number of studies support a critical role for the central melanocortin system in the pathogenesis of cachexia of chronic disease. Mice with impaired central melanocortin signaling due to targeted deletion of the MC4R (MC4RKO) are resistant to the development of cachexia in models of lipopolysaccharide (LPS) sepsis (Marks et al. 2001), cancer (Marks et al. 2001), and acute renal failure (Cheung et al. 2005). Pharmacologic blockade of MC4R signaling with AGRP has been shown to attenuate cachexia in a murine model of cancer cachexia (Joppa et al. 2007). Ghrelin, a gut-derived peptide agonist for the $\mathrm{GH}$ secretagogue receptor that also potently inhibits central melanocortin signaling by increasing Agrp mRNA expression (Kamegai et al. 2000) and hyperpolarizing POMC neurons (Cowley et al. 2003), has been shown to attenuate the development of cachexia in rat models of CHF (Nagaya et al. 2001). Collectively, these data support a direct role for the central melanocortin system in the pathogenesis of cardiac cachexia and suggest that blockade of central melanocortin signaling may represent a potential therapy for cardiac cachexia. There is an important drawback to these studies in that the duration of the inflammatory insult was either acute (e.g. LPS injection) or subacute (e.g. cancer models), and it remains unclear whether melanocortin blockade is a realistic therapeutic target for diseases with a more indolent course (e.g. congestive heart failure, COPD).

In the present paper, we show that chronic blockade of central melanocortin signaling using genetic and pharmacologic techniques is able to attenuate the metabolic manifestations of cardiac cachexia in murine and rat models of heart failure. To our knowledge, these studies are the first to reveal a key role for the central melanocortin system in the pathogenesis of cardiac cachexia, and demonstrate the effectiveness of prolonged antagonism of melanocortin signaling in attenuating cachexia in an animal model of chronic illness.

\section{Materials and Methods}

\section{Animals}

Male wild-type (WT) C57BL/6J mice (6-8 months of age; Jackson Laboratory), MC4RKO mice (6-8 months of age; raised in $\mathrm{C} 57 \mathrm{BL} / 6 \mathrm{~J}$ background), and male Wistar rats (40-50 g; Charles River Laboratories, Wilmington, MA, USA) were maintained on a normal $12 \mathrm{~h}$ light:12 h darkness cycle with ad libitum access to food (Purina rodent diet 5001; Purina Mills) and water. Experiments were conducted in accordance with the NIH Guide for the Care and Use of Laboratory Animals, and approved by the Animal Care and Use Committees of Oregon Health and Science University.

\section{Murine myocardial infarction surgeries}

Prior to the surgeries, mice were divided into four groups: WT sham-operated (WT-Sham, $n=22$ ), WT myocardial infarcted (WT-MI, $n=23$ ), MC4RKO sham-operated (MC4-Sham, $n=19$ ), and MC4RKO MI (MC4-MI, $n=18$ ). Overall surgical mortality was $14 \%$ for WT-Sham group, 35\% for WT-MI group, 11\% for MC4-Sham group, and 39\% for MC4-MI group. Permanent myocardial infarcts were produced in WT and MC4RKO mice by ligation of the anterior descending branch of the left coronary artery as previously described (Gould et al. 2002). Briefly, mice were anesthetized with i.p. pentobarbital sodium $(4 \mathrm{mg} / \mathrm{ml}$, $14 \mu \mathrm{l} / \mathrm{g}$ body weight (BW), Nembutal, Abbott Laboratories), placed in a supine position, and intubated. Mice were ventilated with a mixture of $100 \%$ oxygen and room air with a mechanical rodent Mini-Vent ventilator $(100$ cycles $/ \mathrm{min}$, Harvard Apparatus, Holliston, MA, USA). Ventilator stroke volume was adjusted to fully inflate but not overexpand the lungs. A lateral sternotomy was performed, exposing the anterior surface of the heart. The anterior descending branch of the left main coronary artery (LAD) was ligated at a position $\sim 1 \mathrm{~mm}$ from the tip of the normally positioned left auricle with a 7-0 polypropylene monofilament suture (Prolene, Ethicon, Somerville, NJ, USA). The ligature was not removed after placement. Sham operations were created by passing the suture under the coronary artery at the position used for ligation without ever constricting the artery. After chest closure, mice were recovered in a sternal position, warmed, and provided with $100 \%$ oxygen by nose cone. Post-operative analgesia was extended with a single s.c. injection of $2.5 \mathrm{mg} / \mathrm{kg}$ buprenorphine (Buprenex, Reckitt and Colman Pharmaceuticals; Richmond, VA, USA). Mice must have survived 8 weeks after surgery to be included in the body composition and indirect calorimetry studies.

\section{Murine body composition}

Body composition was determined before infarction surgeries and the end of the experiment by dual-energy X-ray absorptiometry (DEXA, PIXImus mouse densitometer, 
MEC Lunar Corp., Minster, OH, USA). The instrument was calibrated at the start of each recording session with a murine calibration standard. All animals were fasted for $12 \mathrm{~h}$ before DEXA analysis to minimize the effect of ingested food on the DEXA analysis. Individual organ weights (including heart, liver, lung, and spleen) were measured at necropsy at the end of the final DEXA scan.

\section{Murine indirect calorimetry}

Oxygen consumption $\left(\mathrm{VO}_{2}\right)$ was determined by indirect calorimetry (Oxymax, Columbus Instruments, Columbus, $\mathrm{OH}$, USA). Mice were housed in separate chambers at $24 \pm 1{ }^{\circ} \mathrm{C}$. Mice were first acclimatized to the chambers for 2 days prior to conducting the study. Measurements were recorded for $4-8 \mathrm{~h}$ during the middle of the light cycle (1100-1600 h). Samples were recorded every 3 min with the room air reference taken every $30 \mathrm{~min}$ and the air flow to chambers $500 \mathrm{ml} / \mathrm{min}$. Basal oxygen consumption was determined for individual curves as the average of the lowest plateau regions corresponding to resting periods. Total oxygen consumption was the result of all samples recorded corresponding to periods of movement as well as inactivity.

\section{Rat aortic banding surgeries}

Male Wistar rats $(n=40)$ were anesthetized by i.p. injection of pentobarbital sodium $(60 \mathrm{mg} / \mathrm{kg} \mathrm{BW})$. Rats were ventilated with a mixture of $100 \%$ oxygen and room air by mechanical ventilation at a tidal volume of $320 \mu \mathrm{l}$ and a frequency of $120 \mathrm{bpm}$ (MiniVent, Harvard Apparatus). Aortic stenosis $(n=22)$ was induced via a left thoracic incision by banding the ascending aorta with titanium clips (Weck Atrauclip, $0.6 \mathrm{~mm}$ internal diameter) as previously described (HeliesToussaint et al. 2005). The Sham group consisted of shamoperated rats $(n=18)$ prepared by a similar surgical treatment without placement of the clip. After chest closure, rats were recovered in a sternal position, warmed, and provided with $100 \%$ oxygen by nose cone. Post-operative analgesia was extended with a single s.c. injection of $2.5 \mathrm{mg} / \mathrm{kg}$ buprenorphine. Surgical mortality was $18 \%$ for banded rats and $11 \%$ for sham-operated rats. The rats were group housed until the sham-operated rats reached an average weight of $200 \mathrm{~g}$, at which time both sham-operated and banded rats were separated into individual cages for food intake and BW measurement studies.

\section{Rat i.c.v. cannulation and injections}

Cannulation implantation was performed when the average weight of the sham-operated rats reached $300 \mathrm{~g}$. Sham- and aortic-banded rats were anesthetized as described above and placed in a stereotaxic apparatus (Cartesian Research, Inc., Sandy, OR, USA). A sterile guide cannula with obturator stylet was implanted $1 \mathrm{~mm}$ lateral to bregma, $1.5 \mathrm{~mm}$ posterior to bregma, and $3.6 \mathrm{~mm}$ below the surface of the skull.
The cannula was then fixed in place with dental cement. After surgery, the rats were divided into four groups: sham-operated, artificial cerebrospinal fluid (aCSF)-treated (Sham-aCSF, $n=8$ ); sham-operated, AGRP treated (Sham-AGRP, $n=8$ ); banded, aCSF-treated (Band-aCSF, $n=9$ ); and banded, AGRP-treated (Band-AGRP, $n=9$ ), and remained individually housed and allowed 5 days to recover before receiving their first DEXA scan and beginning the treatment period. During recovery, the rats were handled daily and administered $5 \mu \mathrm{l}$ i.c.v. injections of commercial aCSF (Harvard Apparatus) daily. During the 2 -week treatment period, animals received $5 \mu \mathrm{l}$ injections of either aCSF or 1 nmol AGRP (Phoenix Pharmaceuticals, Burlingame, CA, USA) dissolved in $5 \mu \mathrm{l}$ aCSF once every $48 \mathrm{~h}$ for a total of eight injections. One BandAGRP, two Band-aCSF, and two Sham-aCSF rats lost their cannulas prior to the completion of the 2 -week treatment period, and were excluded from the final analysis.

\section{Rat body composition}

Body composition was determined before and after the 2-week AGRP or aCSF treatment period by DEXA (Hologic QDR Discovery A Densitometer) at the OHSU General Clinical Research Center Body Composition core. All animals were fasted for $12 \mathrm{~h}$ before DEXA analysis to minimize the effect of ingested food on the DEXA analysis. Individual organ weights were measured at necropsy at the end of the final DEXA scan. Feed efficiency was calculated by dividing the total weight gained by the total amount of food consumed over the 14-day treatment period. The ratio of lean body mass (LBM) to total body water was determined using quantitative magnetic resonance (EchoMRI, Houston, TX, USA) by the Cincinnati Mouse Metabolic Phenotyping Center as previously described (Tinsley et al. 2004).

\section{Organ necropsy}

A macroscopic necropsy was done for each animal at the time of killing. The heart was removed, the atria and great vessels were removed, and the combined weight of the ventricles and septum was weighed. The liver and kidneys were also removed and weighed. Rat brains were removed, and hypothalamic blocks were dissected out and stored in RNAlater (Ambion, Inc., Austin, TX, USA) at $-80^{\circ} \mathrm{C}$.

\section{$R N A$ preparation and reverse transcription-PCR}

Total RNA was extracted from mice and rat hypothalamic blocks and rat calf muscle using Qiagen RNeasy kits (Qiagen, Inc). DNA was removed from total RNA using RNase-free DNase (Qiagen Inc). Reverse transcription (RT) reactions were prepared using a TaqMan RT Kit (Applied Biosystems, Inc., Foster City, CA, USA).

Real-time RT-PCR was performed on an ABI 7300 Real-Time PCR System using rat-specific primer probe sets obtained from Applied Biosystems. Samples and endogenous 
controls (Eukaryotic 18s rRNA) were run in duplicate to assure repeatability. Auto $C_{\mathrm{t}}$ values were calculated using 7300 RQ Study Software v.1.3 and verified.

\section{Serum TNF- $\alpha$ measurement}

Blood samples were collected in disodium EDTA vacutainers with added aprotinin $(500 \mathrm{KIU} / \mathrm{ml}$ of blood) and centrifuged at $1600 \mathrm{~g}$ for $15 \mathrm{~min}$ at $4{ }^{\circ} \mathrm{C}$. Serum samples were stored at $-80^{\circ} \mathrm{C}$ until assayed for TNF- $\alpha$. Serum TNF- $\alpha$ concentrations were determined using species-specific, commercially available ELISA kits (R\&D Systems, Minneapolis, MN, USA) following the manufacturer's protocol. Radioactive counts were measured using an automated gamma-counter (Beckman LS6500). All samples were run in duplicate.

\section{Statistical analysis}

Data are expressed as mean \pm S.E.M. for each group. Statistical analysis was performed using SPSS (v. 14.0; Chicago, IL, USA) and Prism (v. 5.0) software. Comparisons of data between the four groups were made with one-way ANOVA, followed by a post-hoc analysis using a Bonferroni multiple comparison test. Changes during treatment were analyzed with a two-way ANOVA for repeated measures followed by a Bonferroni test. Comparisons between two groups were made by unpaired Student's $t$-test. Survival was calculated by Kaplan-Meier life table estimates. Group survivals were compared by a log-rank test for homogeneity. For all analyses, significance was assigned at the $P<0.05$ level.

\section{Results}

\section{WT mice develop cardiac cachexia following MI}

Eight weeks after surgery, the heart weights (HWs) of WT-MI mice $(n=15)$ were significantly increased compared to WT-Sham mice $(n=19)$ (WT-MI $0.32 \pm 0.01 \mathrm{~g}$ versus WT-Sham $0 \cdot 16 \pm 0 \cdot 01 \mathrm{~g}, P<0 \cdot 001$; Table 1). The HW-to$\mathrm{BW}$ ratio (HW:BW), a well-established index of cardiac hypertrophy, was significantly greater for WT-MI mice compared to WT-Sham mice (WT-MI $13.7 \pm 0.54 \mathrm{~g}$ versus WT-Sham $6 \cdot 39 \pm 0 \cdot 17 \mathrm{~g}, P<0 \cdot 001$; Table 1). The wet lung weights of WT-MI mice were significantly greater than WT-Sham mice (WT-MI $0 \cdot 29 \pm 0.06 \mathrm{~g}$ versus WT-Sham $0 \cdot 21 \pm 0 \cdot 01 \mathrm{~g}, P<0 \cdot 001$; Table 1). Serum concentration of TNF- $\alpha$ was not significantly different between WT-MI and WT-Sham mice (WT-MI $0 \cdot 18 \pm 0.04 \mathrm{pg} / \mathrm{ml}$ versus WT-Sham $0 \cdot 11 \pm 0 \cdot 05 \mathrm{pg} / \mathrm{ml}, P=\mathrm{NS}$ ). WT-MI mice experienced significantly greater mortality compared to WT-Sham mice $(P<0 \cdot 001$, Fig. 1). Of 34 WT-MI mice, 14 (41\%)

Table 1 Characterization of wild-type versus MC4RKO mice. Data are expressed as mean \pm S.E.M.

\begin{tabular}{|c|c|c|c|c|}
\hline & WT-Sham & WT-MI & MC4-Sham & MC4-MI \\
\hline \multicolumn{5}{|l|}{ Body weight (g) } \\
\hline After treatment & $39 \cdot 93 \pm 1 \cdot 37^{\neq}$ & $29 \cdot 77 \pm 1 \cdot 02$ & $51 \cdot 68 \pm 1 \cdot 09^{*, \neq}$ & $52 \cdot 26 \pm 1 \cdot 24^{*, \neq}$ \\
\hline \multicolumn{5}{|l|}{ Food intake (g/day) } \\
\hline Baseline & $4 \cdot 54 \pm 0 \cdot 08$ & $4 \cdot 37 \pm 0 \cdot 11$ & $6 \cdot 75 \pm 0 \cdot 15^{*}$ & $6 \cdot 78 \pm 0 \cdot 17^{*}$ \\
\hline Percentage of body weight & $14 \cdot 81 \pm 0 \cdot 29$ & $14 \cdot 47 \pm 0 \cdot 52$ & $15 \cdot 91 \pm 0.67$ & $15.99 \pm 0.65$ \\
\hline Heart $(\mathrm{g})$ & $0 \cdot 16 \pm 0 \cdot 01$ & $0 \cdot 32 \pm 0 \cdot 01^{\dagger}$ & $0 \cdot 18 \pm 0 \cdot 01$ & $0 \cdot 32 \pm 0 \cdot 01^{\dagger}$ \\
\hline HW:BW ratio (mg/g) & $4 \cdot 13 \pm 0 \cdot 19$ & $10 \cdot 9 \pm 0 \cdot 57^{\dagger}$ & $3 \cdot 53 \pm 0.11$ & $6 \cdot 20 \pm 0 \cdot 19^{*}$ \\
\hline HW:LBM ratio (mg/g) & $6 \cdot 39 \pm 0 \cdot 17$ & $13 \cdot 7 \pm 0 \cdot 54^{\dagger}$ & $5 \cdot 90 \pm 0 \cdot 21$ & $10 \cdot 4 \pm 0 \cdot 35^{*}$ \\
\hline Lung (g) & $0 \cdot 21 \pm 0 \cdot 01$ & $0 \cdot 29 \pm 0 \cdot 06^{+}$ & $0 \cdot 20 \pm 0 \cdot 01$ & $0 \cdot 32 \pm 0 \cdot 02 *$ \\
\hline Lung:BW ratio (mg/g) & $5 \cdot 50 \pm 0 \cdot 29$ & $10 \cdot 05 \pm 0 \cdot 61^{+}$ & $4 \cdot 14 \pm 0 \cdot 18$ & $6 \cdot 05 \pm 0 \cdot 42 *$ \\
\hline Kidney (g) & $0 \cdot 24 \pm 0 \cdot 01$ & $0 \cdot 20 \pm 0 \cdot 01^{+}$ & $0 \cdot 25 \pm 0 \cdot 01^{*}$ & $0 \cdot 23 \pm 0 \cdot 01 *$ \\
\hline Baseline & $22 \cdot 58 \pm 0 \cdot 26$ & $22 \cdot 96 \pm 0 \cdot 39$ & $27 \cdot 06 \pm 0 \cdot 46^{*}$ & $26 \cdot 95 \pm 0 \cdot 78^{*}$ \\
\hline Percentage of body weight & $73 \cdot 34 \pm 1 \cdot 44$ & $76 \cdot 28 \pm 1 \cdot 84$ & $63 \cdot 29 \pm 1 \cdot 16^{*}$ & $63 \cdot 55 \pm 2 \cdot 18^{*}$ \\
\hline After treatment & $25 \cdot 14 \pm 0 \cdot 45^{\neq}$ & $22 \cdot 83 \pm 0 \cdot 41^{\dagger}$ & $30 \cdot 95 \pm 0 \cdot 52^{* \neq} \neq$ & $30 \cdot 70 \pm 0 \cdot 57^{*, \neq}$ \\
\hline Percentage of body weight & $63 \cdot 82 \pm 1 \cdot 63^{\neq}$ & $77 \cdot 54 \pm 1.98^{\dagger}$ & $59 \cdot 61 \pm 0 \cdot 78^{\ddagger}$ & $58 \cdot 88 \pm 1 \cdot 17^{*}$ \\
\hline \multicolumn{5}{|l|}{ Fat mass $(\mathrm{g})$} \\
\hline Baseline & $8 \cdot 27 \pm 0 \cdot 58$ & $7 \cdot 35 \pm 0 \cdot 56$ & $16 \cdot 30 \pm 0 \cdot 82 *$ & $16 \cdot 37 \pm 1 \cdot 08^{*}$ \\
\hline Percentage of body weight & $26 \cdot 72 \pm 1 \cdot 21$ & $23 \cdot 82 \pm 1 \cdot 71$ & $37 \cdot 71 \pm 1 \cdot 11^{*}$ & $38 \cdot 11 \pm 1 \cdot 85^{*}$ \\
\hline After treatment & $14 \cdot 56 \pm 0.99^{\ddagger}$ & $8 \cdot 11 \pm 0 \cdot 98^{\dagger}$ & $20 \cdot 76 \pm 0 \cdot 68^{*, \neq}$ & $21 \cdot 81 \pm 0 \cdot 99^{*, \neq}$ \\
\hline Percentage of body weight & $35 \cdot 16 \pm 1 \cdot 59^{\neq}$ & $27 \cdot 07 \pm 3 \cdot 22^{+}$ & $40 \cdot 01 \pm 0 \cdot 61$ & $41 \cdot 55 \pm 1 \cdot 11^{*}$ \\
\hline
\end{tabular}

WT, wild-type mice; MC4, MC4RKO mice; Sham, sham-operated mice; MI, myocardial infarction. ${ }^{*} P<0 \cdot 05$ versus respective WT group; ${ }^{\dagger} P<0 \cdot 05$ versus respective Sham group; ${ }^{\ddagger} P<0 \cdot 05$ versus baseline. 


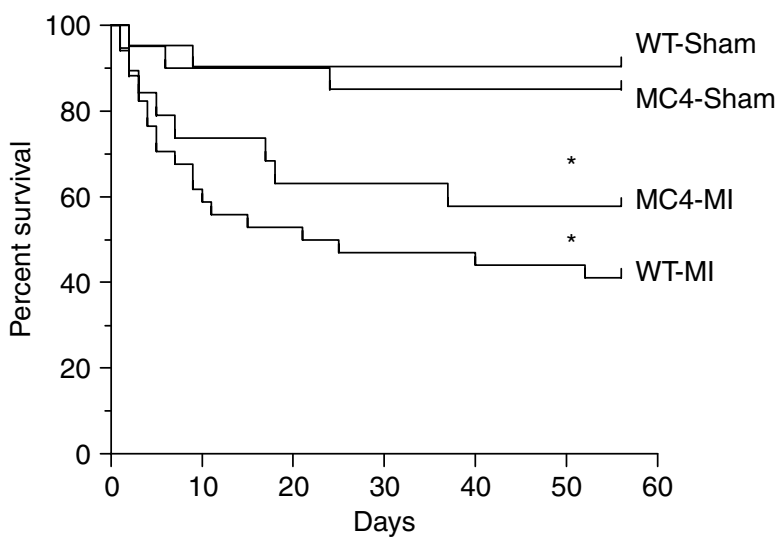

Figure 1 Survival to the end of the 8-week study period after myocardial infarction. WT-Sham, 19 of 21 survived; MC4-Sham, 17 of 20 survived; WT-MI, 14 of 34 survived; MC4-MI, 11 of 19 survived. ${ }^{*} P<0 \cdot 001$ versus WT-Sham and MC4-Sham mice.

survived to the end of the study, compared to 19 of 21 (90\%) WT-Sham mice. Prior to surgery, WT-Sham and WT-MI mice had equal baseline daily food intake (Table 1), but 8 weeks after surgery, the daily food intake of WT-Sham mice was significantly greater than WT-MI mice (WT-Sham $4 \cdot 67$ $\pm 0.07 \mathrm{~g}$ versus WT-MI 3.91 $\pm 0.09 \mathrm{~g}, P<0.01$; Table 1$)$. Daily food intake for WT-Sham mice did not significantly change over the course of the study (Table 1), but significantly declined for WT-MI mice (Table 1). WT-Sham mice gained a significant amount of weight in the period after surgery (Table 1), but WT-MI mice failed to gain weight over the course of the study (Table 1). Significant accumulation of LBM and fat mass after surgery occurred in WT-Sham mice, but not in WT-MI mice (Table 1). The percent increase in LBM per animal over the course of the study was significantly higher for WT-Sham mice compared with WT-MI mice (WT-Sham $11 \cdot 24 \pm 1 \cdot 11 \%$ versus WT-MI $1 \cdot 73 \pm 1 \cdot 27 \%, P<0 \cdot 001$; Fig. 2). Kidney and liver weights were higher in WT-Sham mice compared to WT-MI mice (Table 1) with the difference in kidney weights being significant (WT-Sham $0 \cdot 24 \pm 0 \cdot 01 \mathrm{~g}$ versus WT-MI 0.20 $\pm 0 \cdot 01 \mathrm{~g}, P<0 \cdot 01$; Table 1 ). Eight weeks after surgery, the resting metabolic rate was significantly higher in WT-MI mice $(2826 \pm 78 \mathrm{ml} / \mathrm{kg}$ per h) compared to WT-Sham mice (2371 $\pm 59 \mathrm{ml} / \mathrm{kg}$ per h; $P<0 \cdot 001$; Fig. 3).

\section{MC4RKO mice resist cardiac cachexia following MI}

To test the role of the MC4R in the pathogenesis of cardiac cachexia, we performed sham (MC4-Sham) and MI (MC4-MI) surgeries in MC4RKO mice. Eight weeks after surgery, the HWs of MC4-MI mice $(n=11)$ were significantly increased compared with MC4-Sham mice $(n=17)$ (MC4-MI $0 \cdot 32 \pm 0 \cdot 01 \mathrm{~g}$ versus MC4-Sham $0 \cdot 18 \pm 0 \cdot 01 \mathrm{~g}$, $P<0.001$; Table 1$)$, and these values were not different compared to their respective WT-Sham and WT-MI groups $(P=\mathrm{NS}$; Table 1$)$. The HW:BW ratio was significantly greater for MC4-MI mice compared with MC4-Sham mice

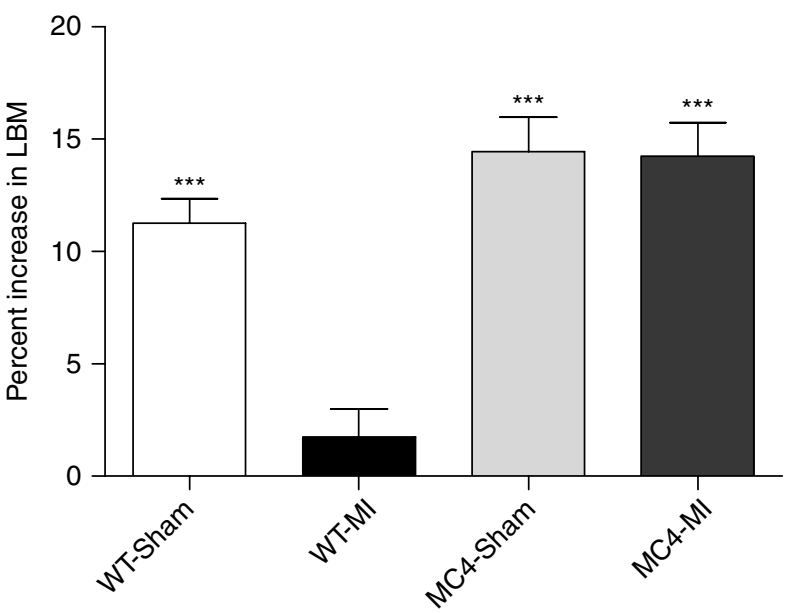

Figure 2 MC4RKO mice resist cardiac cachexia and increase LBM accumulation after myocardial infarction. Data are mean \pm s.E.M. $* * * P<0 \cdot 001$ versus WT-MI.

(MC4-MI 6.20 $\pm 0 \cdot 19 \mathrm{~g}$ versus MC4-Sham $3 \cdot 53 \pm 0 \cdot 11 \mathrm{~g}$, $P<0 \cdot 001$; Table 1). Wet lung weights of MC4-MI mice were significantly increased compared with MC4-Sham mice (MC4-MI $0 \cdot 32 \pm 0 \cdot 02 \mathrm{~g}$ versus MC4-Sham $0 \cdot 20 \pm 0 \cdot 01 \mathrm{~g}$, $P<0 \cdot 001$; Table 1), and these values were not different compared to their respective WT-Sham and WT-MI groups $(P=$ NS; Table 1). Serum concentrations of TNF- $\alpha$ were not significantly different between MC4-MI and MC4-Sham mice (MC4-MI 0.19 $\pm 0.09 \mathrm{pg} / \mathrm{ml}$ versus MC4-Sham $0 \cdot 10 \pm 0 \cdot 07 \mathrm{pg} / \mathrm{ml}, \quad P=\mathrm{NS})$, and were not significantly different compared to WT-MI and WT-Sham mice. MC4-MI mice experienced significantly greater mortality compared with MC4-Sham mice $(P<0 \cdot 001$, Fig. 1$)$. Of 19 MC4-MI mice, $11(58 \%)$ survived to the end of the study,

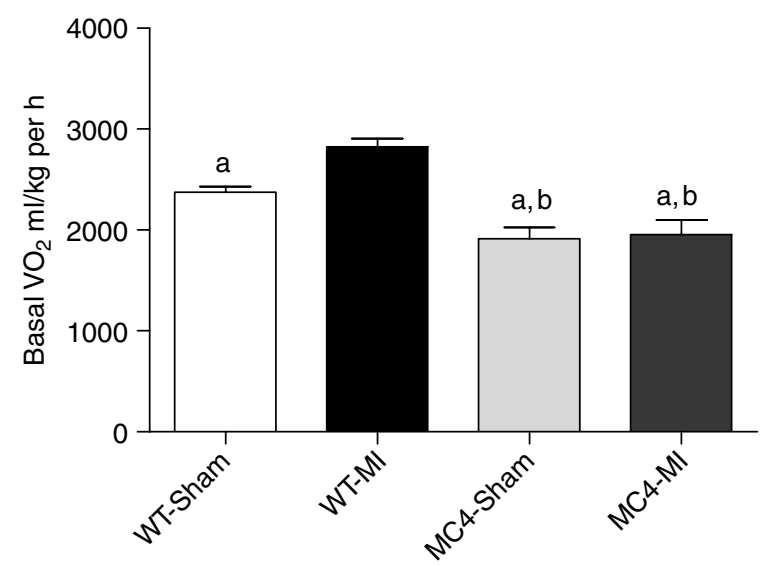

Figure $3 \mathrm{MC} 4 \mathrm{RKO}$ resist an increase in energy expenditure in response to $\mathrm{CHF}$. Basal oxygen consumption during the light phase $(0900-1700 \mathrm{~h})$ is increased in WT-MI mice $(n=15)$ compared with WT-Sham mice $(n=19)$. Basal oxygen consumption is not increased in MC4-MI mice $(n=8)$ compared with MC4-Sham mice $(n=10)$. Data are mean \pm s.E.M. ${ }^{\text {a }} P<0 \cdot 001$ versus WT-MI, ${ }^{\text {b }} P<0 \cdot 05$ versus WT-Sham. 
compared with 17 of 20 (85\%) MC4-Sham mice. A greater percentage of MC4-MI mice survived to 8 weeks after surgery compared with WT-MI mice (58 vs $41 \%$ ), but this difference failed to reach statistical significance in this study. Prior to surgery, MC4-Sham and MC4-MI mice had equal baseline daily food intake (Table 1), and the daily food intake for both groups did not significantly change over the course of the study (Table 1). Both MC4-Sham mice and MC4-MI mice gained significant and comparable amounts of $\mathrm{BW}$, LBM, and fat mass over the course of the study (Table 1). There was no difference in the percent increase in LBM (Fig. 2) and fat mass (Table 1) per animal over the course of the study for MC4-Sham mice compared with MC4-MI mice, and both groups were significantly higher compared to WT-Sham mice (Fig. 2). At necropsy, the kidney weights and liver weights were comparable between MC4-Sham and MC4-MI mice (Table 1). There was no difference in the resting metabolic rate of MC4-MI mice $(1952 \pm 146 \cdot 7 \mathrm{ml} / \mathrm{kg}$ per h) compared with MC4-Sham mice $(1918 \pm 108 \mathrm{ml} / \mathrm{kg}$ per h; $P=$ NS; Fig. 2).

AGRP administration reverses cardiac cachexia in rats with pressure overload cardiac hypertrophy

We next examined the effect of i.c.v. AGRP administration for 2 weeks to reverse cardiac cachexia in a rat model of CHF induced by aortic banding. Prior to being divided into aCSF and AGRP treatment groups, rats receiving sham operations $(n=14)$ were significantly larger than their banded $(n=15)$ littermates (Sham 294.5+3.27 g versus Band 266.6 +5.03 g; $P<0.001$ ), and had higher daily food intake (Sham 25.50 $+0.52 \mathrm{~g}$ versus Band $19 \cdot 67+0.82 \mathrm{~g} ; \quad P<0.001)$. ShamaCSF, Sham-AGRP, and Band-AGRP rats gained significant amounts of $\mathrm{BW}$ over the 2 -week treatment period, but BandaCSF rats failed to significantly gain weight (Table 2 ). Weight gain, LBM, fat mass accumulation, and kidney weights and liver weights were significantly increased by AGRP treatment in banded rats (Band-aCSF versus Band-AGRP; Table 2). The percent increase in LBM per animal over the course of the study was higher in Sham-aCSF rats compared to BandaCSF rats (Sham-aCSF $11 \cdot 62 \pm 2 \cdot 04 \%$ versus Band-aCSF $3 \cdot 39 \pm 2 \cdot 50 \%, P<0 \cdot 05$; Fig. 4). AGRP treatment significantly increased LBM per animal in banded rats when expressed both as a percent of baseline (Band-aCSF 3.39 $\pm 2 \cdot 50 \%$ versus Band-AGRP $17 \cdot 90 \pm 2 \cdot 29 \%, P<0 \cdot 001$; Fig. 4) and when expressed as absolute grams of tissue (BandaCSF $7 \cdot 12 \pm 5.57 \mathrm{~g}$ versus Band-AGRP $52 \cdot 34 \pm 5.96 \mathrm{~g}$; $P<0 \cdot 0001)$. Feed efficiency was significantly decreased in banded rats (Sham-aCSF versus Band-aCSF; Table 2), and significantly increased by AGRP (Band-aCSF versus BandAGRP; Table 2). The heart and wet lung weights of banded rats were significantly increased compared with sham-operated

Table 2 Characterization of sham versus banded rats. Data are expressed as mean \pm S.E.M.

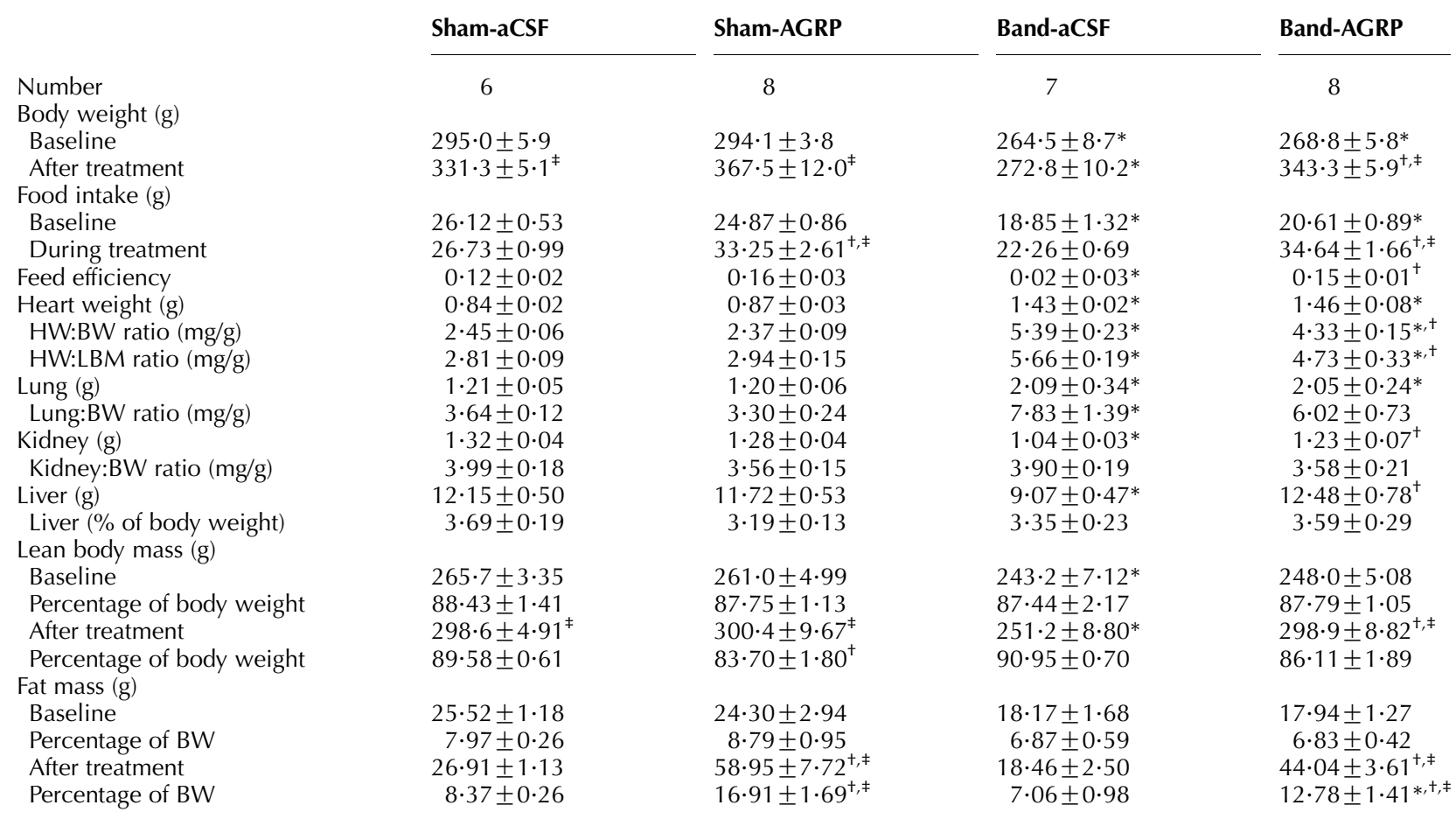

Sham, sham-operated rats; Band, aortic-banded rats; aCSF, i.c.v. aCSF treatment; AGRP, i.c.v. AGRP treatment; BW, body weight. *P<0.05 versus respective sham group; ${ }^{+} P<0.05$ versus respective aCSF group; ${ }^{\ddagger} P<0 \cdot 05$ versus baseline. 


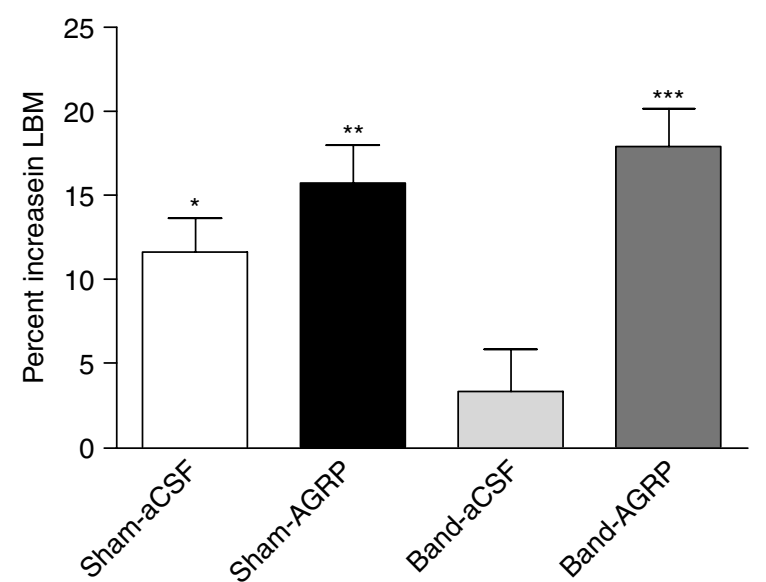

Figure 4 Effect of AGRP versus aCSF administration on the accumulation of LBM in sham-operated and aortic-banded rats. Data are mean \pm s.E.M. ${ }^{*} P<0 \cdot 05$ versus Band-aCSF; ${ }^{* *} P<0 \cdot 01$ versus Band-aCSF; ${ }^{* * *} P<0 \cdot 001$ versus Band-aCSF.

rats (Table 2), but there was no significant difference in heart and wet lung weights between Band-aCSF and Band-AGRP animals. Serum concentrations of TNF- $\alpha$ were not significantly different between banded rats and sham-operated rats (banded $0.21 \pm 0.08 \mathrm{pg} / \mathrm{ml}$ versus sham-operated $0 \cdot 14 \pm 0 \cdot 09 \mathrm{pg} / \mathrm{ml}, P=\mathrm{NS})$. Band-aCSF rats had kidney and liver weights that were significantly decreased compared to Sham-aCSF rats (Table 2). AGRP treatment significantly increased kidney and liver weights in banded rats (Band-aCSF versus Band-AGRP; Table 2), and the final kidney and liver weights of Band-AGRP rats were not different compared to Sham-aCSF rats (Table 2). No significant differences in the hypothalamic mRNA expression of Pomc, Agrp, neuropeptide $\mathrm{Y}, I l 1 \beta$, Il1r, prohormone convertase 1 (Pc1 or Pcsk1 as listed in the MGI Database), and Pc2 (Pcsk2) were found between Sham-aCSF, Sham-AGRP, Band-aCSF, and Band-AGRP rats. The survival rate for both banded and sham-operated rats that received either saline or AGRP for 2 weeks was $100 \%$ over the 2 -week injection period.

\section{Discussion}

In agreement with results from a previously published study (Gould et al. 2002), WT mice that received permanent LAD ligation surgeries developed physiologic abnormalities consistent with a cachectic state including weight loss, reduced LBM, reduced fat mass accumulation, and organ atrophy. In contrast, MC4-MI mice as a group did not become cachectic as shown by their normal accumulation of LBM, fat mass, and overall normal gross organ weights as compared to MC4-Sham mice. These results argue that in addition to attenuating cachexia in the acute illness, loss of MC4R signaling is also effective in providing prolonged protection against the development of cachexia in the setting of a chronic illness. In comparing the survival of MC4-MI mice to WT-MI mice after surgery, we observed that a greater percentage of MC4-MI mice survived compared to WT-MI mice, though this difference did not achieve statistical significance in this study. However, these results do suggest that melanocortin blockade may confer a survival advantage in the setting of heart failure, and that additional experiments with larger groups of animals may be warranted to further investigate this possibility.

Although a direct measurement of cardiac function via Doppler ultrasound was not performed in this study, both WT-MI and MC4-MI mice achieved HW:BW ratios that were significantly greater than their respective sham controls. Previous studies have demonstrated that mice with elevated HW:BW ratios similar to those measured in our study are consistent with the animals being in heart failure (Nahrendorf et al. 2003, Shioura et al. 2007, 2008). In addition, the increased wet lung weights of both WT-MI and MC4-MI suggest that these animals had developed significant pulmonary edema, providing further support that these animals were in a state of heart failure. One concern at the beginning of the study is that loss of MC4R signaling could potentially alter the sensitivity of the hearts to the effects of the MI procedure. Ending HWs for sham-operated mice (WT-Sham versus MC4-Sham) and MI mice (WT-MI versus MC4-MI) were not different from each other. These results argue against the loss of MC4R signaling providing a protective effect against ischemic damage as a result of the MI procedure, or altering the subsequent development of cardiohypertrophy.

To test the ability of specific blockade of central MC4R signaling to attenuate cardiac cachexia, we utilized a pharmacological approach by administering multiple i.c.v. injections of AGRP to animals that had developed cardiac cachexia. For this experiment, we decided to use a rat model of left ventricular pressure overload hypertrophy, a wellcharacterized model of cardiac cachexia (Helies-Toussaint et al. 2005). At the beginning of the treatment period, banded rats were hypophagic, and had reduced BWs, LBM, and fat mass accumulation compared to sham-operated rats. AGRP treatment in banded rats was effective in attenuating the cachectic state as evidenced by the significant increases in food intake, LBM, and fat mass accumulation while receiving treatment. Feed efficiency, a measurement of weight gain per unit of food consumed, was significantly increased by banding and was reversed by AGRP treatment. These results support the conclusion that the observed increases in weight, LBM, and fat mass in Band-AGRP rats were not only due to AGRP increasing food intake, but also due to AGRP decreasing the increased metabolic rate that results from the banding procedure. Although our current study was not designed to measure the effect of AGRP treatment on morbidity in banded rats, we observed during the treatment period that Band-AGRP rats were more active and better groomed compared to Band-aCSF rats. Indirect calorimetry measurements may have provided some information regarding the relative activity of the groups, but these measurements were 
not performed due to the inability of the murine chambers to be used for adult rats. Final gross HWs for sham-operated rats (Sham-aCSF versus Sham-AGRP) and banded rats (Band-aCSF versus Band-AGRP) were not different from each other respectively, demonstrating that central AGRP treatment did not promote cardiohypertrophy in sham rats, nor prevent cardiohypertrophy from occurring in banded rats. Collectively, these results reveal that blockade of central melanocortin signaling with AGRP is both sufficient and successful in attenuating cardiac cachexia in the aortic-banded rat model of pressure overload cardiac hypertrophy.

In human patients with CHF, elevated levels of the proinflammatory cytokine $\mathrm{TNF} \alpha$ have been measured in patients that have developed cardiac cachexia (Anker \& Sharma 2002), and have been correlated with worsening cachexia (Dutka et al. 1993, Anker et al. 2004). In contrast to these human studies, we did not find significant increases in serum $\mathrm{TNF} \alpha$ in either our mouse or rat models of cardiac cachexia. Similar to our results, the absence of a significant rise in serum TNF $\alpha$ in a murine model of MI-induced CHF has also been reported by another group (Schulze et al. 2003), raising the intriguing possibility that this may be representative of a species-specific difference between humans and rodents in the peripheral inflammatory response to CHF. In the absence of a robust peripheral TNF- $\alpha$ response, the question is then raised how else could inflammatory signals be relayed to the brain to increase central melanocortin signaling? One alternative hypothesis is that afferent fibers from autonomic nerves that innervate the heart relay stimulatory signals to the brain from the chronically inflamed, failing heart. Indeed, the ability of autonomic fibers that innervate the heart to stimulate the production of proinflammatory cytokines in the hypothalamus has already been demonstrated in a murine model of acute MI (Francis et al. 2004). Combined with recent data that have clearly established that central paracrine production of cytokines within the brain (by resident microglia) is both necessary and sufficient for the production of a cachectic state (Ogimoto et al. 2006, Wisse et al. 2007), it is possible in rodents that the development of cachexia in the setting of CHF is independent of a significant rise in peripheral proinflammatory cytokines. Although we found no significant differences in the hypothalamic expression of either IL1 $\beta$ or IL1R, there remains many additional proinflammatory cytokines capable of promoting a cachectic state including TNF- $\alpha$, IL6, and leukemia inhibitory factor whose expression in the hypothalamus in the setting of CHF remains to be investigated.

In addition to being expressed in the brain, $M c 4 r$ mRNA expression has also been demonstrated in peripheral systems, including the heart and lung (Mountjoy et al. 2003). This observation raises the possibility that increased peripheral MC4R signaling may be contributing to the pathogenesis of cardiac cachexia, and that blockade of this signaling as would occur with MC4RKO mice might contribute to the prevention of cardiac cachexia. Phenotypic analysis of MC4RKO mice has failed to reveal the development of any cardiovascular abnormalities, and humans with defective $M C 4 R$ genes have not been reported to suffer from increased cardiovascular problems independent of those associated with their increased risk of developing the metabolic syndrome. Our observation in this study that central injections of AGRP, a neuropeptide that is incapable of crossing the blood-brain barrier, are able to attenuate cardiac cachexia in our rat model of CHF strongly suggests that central MC4R signaling is the principle driving force for the cachectic state, and that the relative contribution of MC4R signaling from the periphery is likely minor. Although the functional role of MC4R signaling in the cardiorespiratory system remains to be elucidated, there is evidence that the MC3R plays a protective role in acute and delayed heart reperfusion injury (Mioni et al. 2003, Getting et al. 2004). Activation of MC3R led to reduced myocardial cytokine production and myeloperoxidase activity, and these effects were prevented by the mixed MC3/4R antagonist SHU9119, but not by the selective MC4R antagonist HS204 (Getting et al. 2004).

An interesting observation in our study was that the blockade of central melanocortin signaling resulted in significantly increased adiposity in rats with cardiac cachexia due to left ventricular pressure overload hypertrophy. Repeated injections of AGRP have been shown to cause hyperphagia and obesity in healthy rats and mice (Small et al. 2001). However, this is the first study that shows that these results also occur in rodents that have cardiac cachexia at baseline. Although increased adiposity is a risk factor for cardiovascular disease and the subsequent development of CHF (Kenchaiah et al. 2002), data suggest that once CHF has developed, overweight and obese patients paradoxically have better prognosis and lower mortality risks than lean patients (Curtis et al. 2005). Band-AGRP rats had significantly more fat mass than Sham-aCSF rats (Table 2), but unlike MC4RKO mice where the increased adiposity has the adverse outcome of pronounced liver steatosis, we did not observe liver steatosis in the Band-AGRP rats.

In summary, our data suggest that the central melanocortin system, a neural integrator of hormone and cytokine signaling that regulates feeding behavior, linear growth, and metabolic rate, may play a key role in the pathogenesis of cardiac cachexia.

The ability of genetic and pharmacologic blockade of melanocortin signaling to increase both LBM and fat mass in rodent models of $\mathrm{CHF}$ suggests that compounds that selectively antagonize central melanocortin signaling may have important pharmacotherapeutic benefits for cachectic CHF patients.

\section{Declaration of interest}

DLM is a consultant for IPSEN, Inc., a company that may have a commercial interest in the results of this research and technology. This potential conflict has been reviewed and managed by the OHSU Conflict of Interest in Research Committee and the Integrity Program Oversight Council. 


\section{Funding}

This work was supported by a National Institute of Diabetes and Digestive and Kidney Diseases Grant DK70333 and an American Heart Association Predoctoral Fellowship 0515502Z.

\section{References}

Anker SD \& Sharma R 2002 The syndrome of cardiac cachexia. International Journal of Cardiology 85 51-66.

Anker SD, Ponikowski P, Varney S, Chua TP, Clark AL, Webb-Peploe KM, Harrington D, Kox WJ, Poole-Wilson PA \& Coats AJ 1997 Wasting as independent risk factor for mortality in chronic heart failure. Lancet 349 1050-1053.

Anker SD, Ponikowski PP, Clark AL, Leyva F, Rauchhaus M, Kemp M, Teixeira MM, Hellewell PG, Hooper J, Poole-Wilson PA et al. 1999 Cytokines and neurohormones relating to body composition alterations in the wasting syndrome of chronic heart failure. European Heart Journal 20 683-693.

Anker S, Steinborn W \& Strassburg S 2004 Cardiac cachexia. Annals of Medicine 36 518-529.

Baskin DG, Hahn TM \& Schwartz MW 1999 Leptin sensitive neurons in the hypothalamus. Hormone and Metabolic Research 31 345-350.

Campbell IL 1998 Transgenic mice and cytokine actions in the brain: bridging the gap between structural and functional neuropathology. Brain Research. Brain Research Reviews 26 327-336.

Cheung W, Yu PX, Little BM, Cone RD, Marks DL \& Mak RH 2005 Role of leptin and melanocortin signaling in uremia-associated cachexia. Journal of Clinical Investigation 115 1659-1665.

Cone RD 2005 Anatomy and regulation of the central melanocortin system. Nature Neuroscience 8 571-578.

Cone RD, Cowley MA, Butler AA, Fan W, Marks DL \& Low MJ 2001 The arcuate nucleus as a conduit for diverse signals relevant to energy homeostasis. International Journal of Obesity and Related Metabolic Disorders 25 (Suppl 5) S63-S67.

Cowie MR, Wood DA, Coats AJ, Thompson SG, Poole-Wilson PA, Suresh V \& Sutton GC 1999 Incidence and aetiology of heart failure: a populationbased study. European Heart Journal 20 421-428.

Cowley MA, Smith RG, Diano S, Tschop M, Pronchuk N, Grove KL, Strasburger CJ, Bidlingmaier M, Esterman M, Heiman ML et al. 2003 The distribution and mechanism of action of ghrelin in the CNS demonstrates a novel hypothalamic circuit regulating energy homeostasis. Neuron 37 649-661.

Curtis JP, Selter JG, Wang Y, Rathore SS, Jovin IS, Jadbabaie F, Kosiborod M, Portnay EL, Sokol SI, Bader F et al. 2005 The obesity paradox: body mass index and outcomes in patients with heart failure. Archives of Internal Medicine 165 55-61.

Dutka DP, Elborn JS, Delamere F, Shale DJ \& Morris GK 1993 Tumour necrosis factor alpha in severe congestive cardiac failure. British Heart Journal 70 141-143.

Fan W, Boston BA, Kesterson RA, Hruby VJ \& Cone RD 1997 Role of melanocortinergic neurons in feeding and the agouti obesity syndrome. Nature 385 165-168.

Foster AC, Joppa M, Markison S, Gogas KR, Fleck BA, Murphy BJ, Wolff M, Cismowski MJ, Ling N, Goodfellow VS et al. 2003 Body weight regulation by selective MC4 receptor agonists and antagonists. Annals of the New York Academy of Sciences 994 103-110.

Francis J, Chu Y, Johnson AK, Weiss RM \& Felder RB 2004 Acute myocardial infarction induces hypothalamic cytokine synthesis. American Journal of Physiology. Heart and Circulatory Physiology 286 H2264-H2271.

Getting SJ, Di Filippo C, Christian HC, Lam CW, Rossi F, D’Amico M \& Perretti M 2004 MC-3 receptor and the inflammatory mechanisms activated in acute myocardial infarct. Journal of Lenkocyte Biology $\mathbf{7 6}$ 845-853.

Gould KE, Taffet GE, Michael LH, Christie RM, Konkol DL, Pocius JS, Zachariah JP, Chaupin DF, Daniel SL, Sandusky GE Jr et al. 2002 Heart failure and greater infarct expansion in middle-aged mice: a relevant model for postinfarction failure. American Journal of Physiology. Heart and Circulatory Physiology 282 H615-H621.

Helies-Toussaint C, Moinard C, Rasmusen C, Tabbi-Anneni I, Cynober L \& Grynberg A 2005 Aortic banding in rat as a model to investigate malnutrition associated with heart failure. American Journal of Physiology. Regulatory, Integrative and Comparative Physiology 288 R1325-R1331.

Joppa MA, Ling N, Chen C, Gogas KR, Foster AC \& Markison S 2005 Central administration of peptide and small molecule MC4 receptor antagonists induce hyperphagia in mice and attenuate cytokine-induced anorexia. Peptides 26 2294-2301.

Joppa MA, Gogas KR, Foster AC \& Markison S 2007 Central infusion of the melanocortin receptor antagonist agouti-related peptide $(\operatorname{AgRP}(83-132))$ prevents cachexia-related symptoms induced by radiation and colon-26 tumors in mice. Peptides 28 636-642.

Kamegai J, Tamura H, Shimizu T, Ishii S, Sugihara H \& Wakabayashi I 2000 Central effect of ghrelin, an endogenous growth hormone secretagogue, on hypothalamic peptide gene expression. Endocrinology 141 4797-4800.

Kenchaiah S, Evans JC, Levy D, Wilson PW, Benjamin EJ, Larson MG, Kannel WB \& Vasan RS 2002 Obesity and the risk of heart failure. New England Journal of Medicine 347 305-313.

Levine B, Kalman J, Mayer L, Fillit HM \& Packer M 1990 Elevated circulating levels of tumor necrosis factor in severe chronic heart failure. New England Journal of Medicine 323 236-241.

Lloyd-Jones DM, Larson MG, Leip EP, Beiser A, D’Agostino RB, Kannel WB, Murabito JM, Vasan RS, Benjamin EJ \& Levy D 2002 Lifetime risk for developing congestive heart failure: the Framingham Heart Study. Circulation 106 3068-3072.

Marks DL, Ling N \& Cone RD 2001 Role of the central melanocortin system in cachexia. Cancer Research 61 1432-1438.

Mioni C, Giuliani D, Cainazzo MM, Leone S, Bazzani C, Grieco P, Novellino E, Tomasi A, Bertolini A \& Guarini S 2003 Further evidence that melanocortins prevent myocardial reperfusion injury by activating melanocortin MC3 receptors. European Journal of Pharmacology 477 227-234.

Mountjoy KG, Jenny Wu CS, Dumont LM \& Wild JM 2003 Melanocortin-4 receptor messenger ribonucleic acid expression in rat cardiorespiratory, musculoskeletal, and integumentary systems. Endocrinology 144 5488-5496.

Nagaya N, Uematsu M, Kojima M, Ikeda Y, Yoshihara F, Shimizu W, Hosoda H, Hirota Y, Ishida H, Mori H et al. 2001 Chronic administration of ghrelin improves left ventricular dysfunction and attenuates development of cardiac cachexia in rats with heart failure. Circulation 104 1430-1435.

Nahrendorf M, Hiller KH, Hu K, Ertl G, Haase A \& Bauer WR 2003 Cardiac magnetic resonance imaging in small animal models of human heart failure. Medical Image Analysis 7 369-375.

Ogimoto K, Harris MK Jr \& Wisse BE 2006 MyD88 is a key mediator of anorexia, but not weight loss, induced by lipopolysaccharide and interleukin-1 beta. Endocrinology 147 4445-4453.

Ollmann MM, Wilson BD, Yang YK, Kerns JA, Chen Y, Gantz I \& Barsh GS 1997 Antagonism of central melanocortin receptors in vitro and in vivo by agouti-related protein. Science 278 135-138.

Plata-Salaman CR 2001 Cytokines and feeding. International Journal of Obesity and Related Metabolic Disorders 25 (Suppl 5) S48-S52.

Plata-Salaman CR, Sonti G, Borkoski JP, Wilson CD \& French-Mullen JMB 1996 Anorexia induced by chronic central administration of cytokines at estimated pathophysiological concentrations. Physiology and Behavior 60 867-875.

Reyes TM \& Sawchenko PE 2002 Involvement of the arcuate nucleus of the hypothalamus in interleukin-1-induced anorexia. Journal of Neuroscience 22 5091-5099.

Rosamond W, Flegal K, Furie K, Go A, Greenlund K, Haase N, Hailpern SM, Ho M, Howard V, Kissela B et al. 2008 Heart disease and stroke statistics 2008 update: a report from the American Heart Association Statistics Committee and Stroke Statistics Subcommittee. Circulation 117 e25-e146. 
Scarlett JM, Jobst EE, Enriori PJ, Bowe DD, Batra AK, Grant WF, Cowley MA \& Marks DL 2007 Regulation of central melanocortin signaling by interleukin-1 $\beta$. Endocrinology 148 4217-4225.

Scarlett JM, Zhu X, Enriori PJ, Bowe DD, Batra AK, Levasseur PR, Grant WF, Meguid MM, Cowley MA \& Marks DL 2008 Regulation of agouti-related protein messenger ribonucleic acid transcription and peptide secretion by acute and chronic inflammation. Endocrinology 149 $4837-4845$.

Schulze PC, Gielen S, Adams V, Linke A, Mobius-Winkler S, Erbs S, Kratzsch J, Hambrecht R \& Schuler G 2003 Muscular levels of proinflammatory cytokines correlate with a reduced expression of insulinlike growth factor-I in chronic heart failure. Basic Research in Cardiology 98 267-274.

Shioura KM, Geenen DL \& Goldspink PH 2007 Assessment of cardiac function with the pressure-volume conductance system following myocardial infarction in mice. American Journal of Physiology. Heart and Circulatory Physiology 293 H2870-H2877.

Shioura KM, Geenen DL \& Goldspink PH 2008 Sex-related changes in cardiac function following myocardial infarction in mice. American Journal of Physiology. Regulatory, Integrative and Comparative Physiology 295 R528-R534.
Small CJ, Kim MS, Stanley SA, Mitchell JR, Murphy K, Morgan DG, Ghatei MA \& Bloom SR 2001 Effects of chronic central nervous system administration of agouti-related protein in pair-fed animals. Diabetes $\mathbf{5 0}$ 248-254.

Tinsley FC, Taicher GZ \& Heiman ML 2004 Evaluation of a quantitative magnetic resonance method for mouse whole body composition analysis. Obesity Research 12 150-160.

Tisdale MJ 1997 Biology of cachexia. Journal of the National Cancer Institute 89 1763-1773.

Wisse BE, Ogimoto K, Tang J, Harris MK Jr, Raines EW \& Schwartz MW 2007 Evidence that lipopolysaccharide-induced anorexia depends upon central, rather than peripheral, inflammatory signals. Endocrinology 148 $5230-5237$.

Received in final form 15 March 2010

Accepted 6 April 2010

Made available online as an Accepted Preprint

6 April 2010 